\title{
Simultaneous ion beam profile scan using a single laser source
}

\author{
Y. Liu, C. Long, C. Huang, R. Dickson, and A. Aleksandrov \\ Spallation Neutron Source, Oak Ridge National Laboratory, Oak Ridge, Tennessee 37831, USA
}

(Received 7 November 2012; published 28 January 2013)

\begin{abstract}
We report on the world's first experiment of a simultaneous profile scan of the hydrogen ion $\left(\mathrm{H}^{-}\right)$beam using a laser wire system. The system was developed and brought to operational level of application at the superconducting linac of the Spallation Neutron Source accelerator complex. The laser wire profile scanner is based on a photodetachment process and therefore can be conducted on a 1-MW neutron production $\mathrm{H}^{-}$beam in a nonintrusive manner. The new simultaneous profile scanning system allows one to simultaneously measure profiles of the $\mathrm{H}^{-}$beam at nine different locations of the linac with high speed and accuracy, and therefore provides a unique tool for accelerator tuning and physics study. This paper describes the design, optical system and software platform developments, and measurement results of the simultaneous profile scanning system.
\end{abstract}

DOI: 10.1103/PhysRevSTAB.16.012801

PACS numbers: 41.85.Ew, 07.60.-j, 41.75.Cn, 41.85.Qg

\section{INTRODUCTION}

Nonintrusive beam profile and emittance measurements have attracted increasing attention since they have little risk of causing equipment damage and can be conducted under operational particle beam conditions (i.e., particle beams with high beam current, long pulse duration, and/or high repetition rates) [1-9]. In recent years, laser based nonintrusive hydrogen ion $\left(\mathrm{H}^{-}\right)$beam profile/emittance measurement systems have been commissioned at the Spallation Neutron Source (SNS) which is the accelerator based, world's most intense short-pulse neutron scattering facility $[8,9]$. Measurements of the neutron production $\mathrm{H}^{-}$ beam profile and emittance using laser wires have been presented in previous papers. It was demonstrated that the laser wire system has an excellent temporal resolution and was able to measure profiles at different segments of the ion beam's minipulse [10] and thus provides a unique profile study that is unavailable with the technology of conventional profile monitors such as wire scanners.

In this paper we report on our recent developments on the simultaneous profile scan system in the superconducting linac (SCL) of the SNS using the laser wire system. Although laser wire profile monitors have been installed at multiple locations, the profile measurement was performed in a serial manner since a single light source was used. On the other hand, physics study such as the SCL modeling at SNS requires the measurement of $\mathrm{H}^{-}$beam profiles at different locations along the acceleration path and usually such measurement needs to be conducted many times on different accelerator settings. A simultaneous profile measurement would be especially helpful to improve the

Published by the American Physical Society under the terms of the Creative Commons Attribution 3.0 License. Further distribution of this work must maintain attribution to the author(s) and the published article's title, journal citation, and DOI. efficiency and accuracy of the physics studies. We show that through a series of improvements on the optical transfer line, timing control, and scanning software, it is possible to simultaneously measure $\mathrm{H}^{-}$beam profiles at nine different locations along the SCL. The entire measurement process of both horizontal and vertical directions will only take 5 minutes. The measurement was conducted completely in a nonintrusive manner on the neutron production $\mathrm{H}^{-}$beam. Together with the hardware modification, we have also upgraded our user interface to provide a pushbutton style, informative platform to make the laser wire system a truly convenient and useful tool for the accelerator operators and physicists.

\section{EXPERIMENTAL SETUP}

Detailed descriptions of the SNS laser wire system have been given in our previous papers [8,9]. Here we focus on the major modifications and improvements which allow the simultaneous profile scans using a single light source.

\section{A. System outline and measurement principle}

Figure 1 shows a layout of the laser wire system implemented at the SCL of SNS. The 230-meter long SCL is the longest section of the SNS accelerator complex and it accelerates the $\mathrm{H}^{-}$from $186 \mathrm{MeV}$ to $1 \mathrm{GeV}$. The SCL consists of 23 cryomodules with each cryomodule housing three or four superconducting cavities. Currently, a total of nine laser wire stations have been commissioned with the first four stations located after each of the first four cryomodules, the next four stations after cryomodules 12 through 15, and the last station at the end of the SCL. Therefore, the nine profile monitors provide a nonintrusive way of tracking profile changes of the neutron production $\mathrm{H}^{-}$beam along its acceleration path.

A schematic illustration of the laser wire measurement station is shown in the inset box A of Fig. 1. At each 


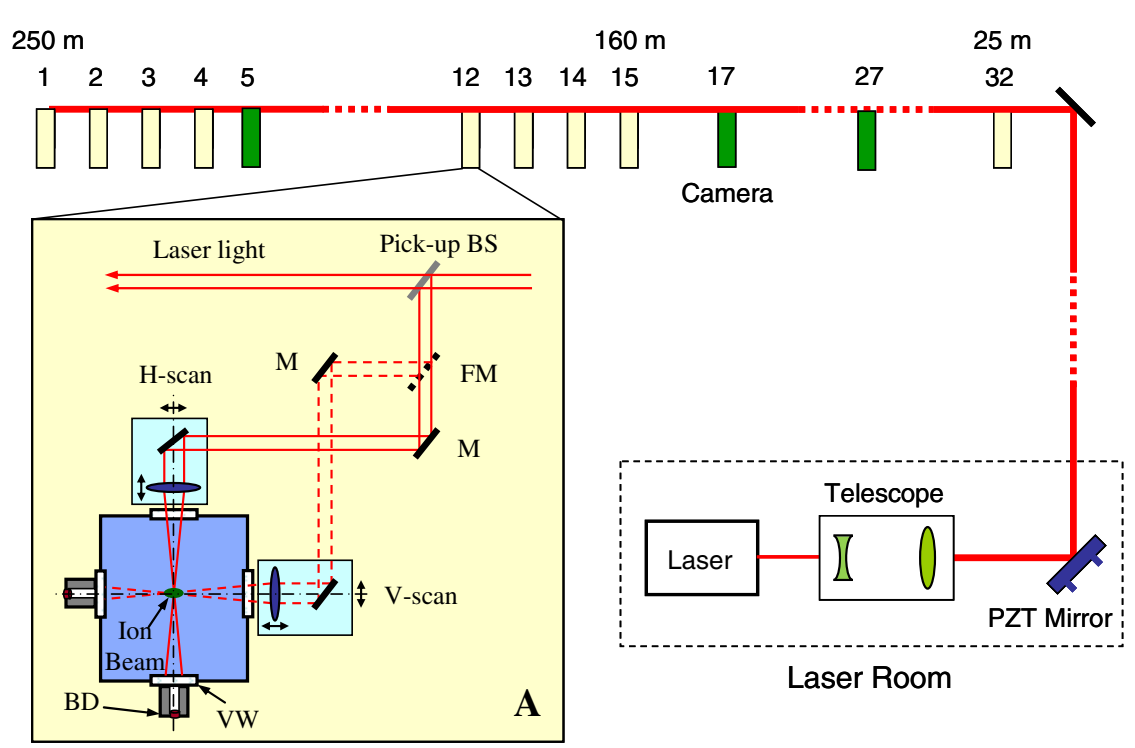

FIG. 1. Outline of the SNS laser wire system. PZT mirror: piezoelectric transducer driven mirror for beam position stabilization. The numbers in the figure indicate the SCL cryomodule and distances are from the laser source. Inset box A: diagram of an individual profile measurement station. BS: beam splitter; M: mirror; FM: flipper mirror; BD: beam dump; VM: vacuum window.

measurement station, a stepper motor driven pickup mirror intercepts the free-space laser transport line (LTL) and redirects the laser light to the measurement box. Inside the measurement box, a motorized flip mirror switches the laser light between the horizontal and vertical scanning axes. The laser beam enters a vacuum chamber through a vacuum window (laser port) and is designed to interact with the ion beam close to the focal point of the laser beam. The laser beam diameter at the interaction point is about $100 \mu \mathrm{m}$. After the interaction, the laser beam passes through the back window of the vacuum and hits the beam dump. A photodiode is installed at the center of the beam dump to receive a small portion of the laser beam. The photodiode output gives an indication signal of laser beam presence. The scanning translation stages have a travel range of $46 \mathrm{~mm}$ with a $\mu \mathrm{m}$ order resolution. Normally a scan step of $0.25 \mathrm{~mm}$ results in a sufficient resolution of profiles [8].

When the $\mathrm{H}^{-}$beam interacts with the laser light, a small portion of the ions illuminated by the laser pulse are ionized to $\mathrm{H}^{0}$ and the liberated electrons are collected by an electron detector. The measurement of the resulting electron density leads to the determination of the negative ion density. By scanning the incident laser beam in horizontal or vertical directions, profiles of the ion beam along the correspondent axis can be obtained.

\section{B. Optical system modifications}

All nine SCL laser wire profile monitors use the same, single Q-switched Nd:YAG laser as a light source. This laser is capable of producing a maximum pulse energy of $1.5 \mathrm{~J}$ with a $7 \mathrm{~ns}$ pulse width and a $30 \mathrm{~Hz}$ pulse rate. The firing of laser pulses is triggered by a clock signal from the accelerator baseline timing system. The laser is located outside the linac tunnel to avoid radiation damage and the laser beam is delivered to SCL through an enclosed freespace LTL. The laser pulse energy is continuously tunable from the millijoule level to over $1 \mathrm{~J}$ by using two sets of half-wave plates and polarization beam splitters installed right after the laser. The actual pulse energy is clamped to $0.5 \mathrm{~J}$ for safety. High beam quality is important for the laser beam because it needs to transport over 250 meters. The laser beam shows a well-defined $\mathrm{TEM}_{00}$ Gaussian mode with an $\mathrm{M}^{2}$ factor estimated to be about 4 and 6 in horizontal and vertical directions, respectively. Single wavelength and narrow line width are required to avoid photodetachment noise induced by the temporal jitter of the laser pulse. A narrowband injection seeder has been recently upgraded to provide a smoother temporal profile and reduce the linewidth to about $100 \mathrm{MHz}$.

To improve the laser beam collimation quality, a large aperture telescope (Special Optics Model 52-71-3.5X$1064 \mathrm{~nm}$ ) was customized to produce a collimated laser beam with a beam diameter of up to $5 \mathrm{~cm}$. The modified collimation setup allows laser beam diameters within $2-3 \mathrm{~cm}$ at all laser wire measurement stations. The laser beam profiles along the SCL are measured with an image sensor. The measured beam profile is then fit with a Gaussian function which gives the beam widths $w_{x}$ and $w_{y}$ at horizontal and vertical directions, respectively. Figure 2 shows the laser beam size (denoted as the area of the beam spot, i.e., $\pi w_{x} w_{y}$ ) measured at three locations along the SCL: CM27, CM17, and CM05 corresponding to the beginning, middle, and end of the SCL. The distances of the above locations from the laser source are 46, 140, and 227 meters, respectively. The beam sizes are plotted in 

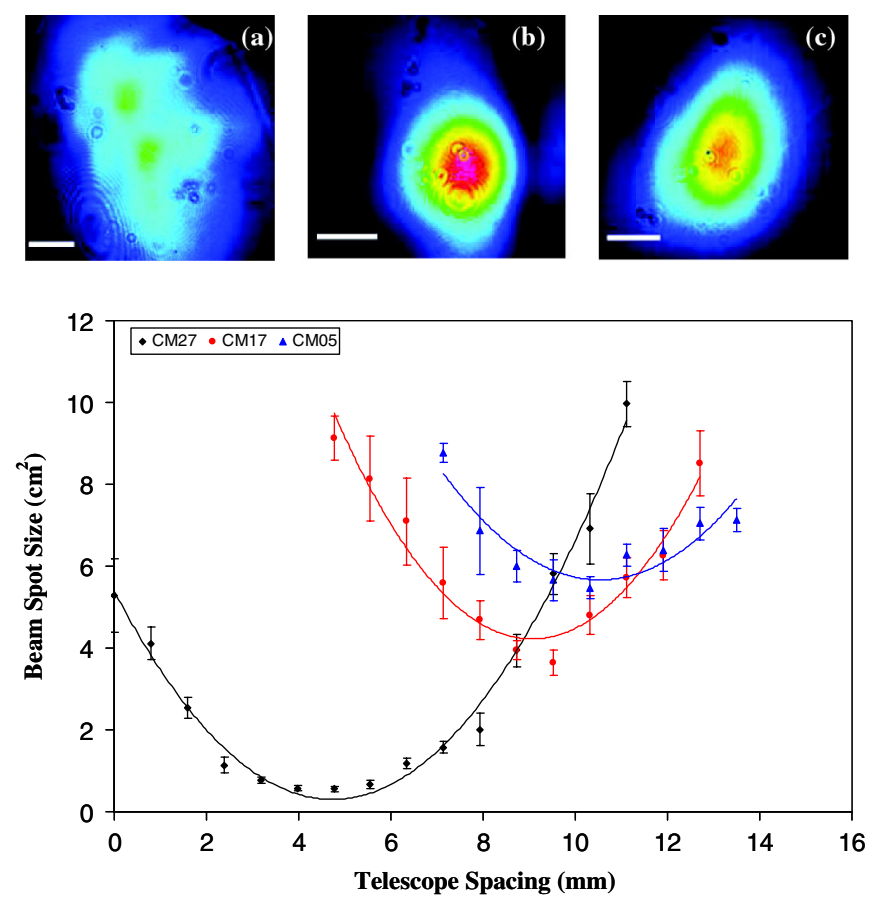

FIG. 2. Laser beam spot sizes measured at three locations of the SCL as a function of the telescope spacing (distance between focusing and defocusing lenses). Inset boxes $a, b$, and c: images of the laser beam spot measured at cryomodule (CM) 27, 17, and 5 , respectively. All scale bars in inset boxes correspond to $1 \mathrm{~cm}$.

Fig. 2 as a function of the telescope spacing. The telescope spacing (distance between focusing and defocusing lenses) is variable from 0 to $16 \mathrm{~mm}$ and is currently set at $\sim 10 \mathrm{~mm}$ to yield the smallest beam size at the CM05 that is close to the end of LTL. At this telescope setting, the corresponding beam images measured at CM27, CM17, and CM05 are shown in the inset boxes a, b, and c, respectively. At the optimum collimation setting, we can expect to have welldefined Gaussian laser beam profiles along the transport line for most of the laser wire stations, i.e., CM1-12. The laser beam profile at CM27 (and similarly at the measurement station CM32) shows a non-Gaussian distribution since the location is far away $(\sim 100 \mathrm{~m})$ from the laser beam focal plane and some near-field feature of the laser beam presents in the profile. However, it should be pointed out that such profile irregularities will not affect the measurement at CM32 since the $\mathrm{H}^{-}$beam interacts with the laser light near the focal point of the laser beam where a well-defined Gaussian distribution can always be assumed.

Except for the last measurement station, all the pickup mirrors in the transport line were switched from the original $5-\mathrm{cm}$ diameter mirrors to $7.5-\mathrm{cm}$ diameter and $5-\mathrm{mm}$ thick beam splitters. Thin optics was chosen to minimize the beam drift during propagation through tilted beam splitters. The optical mount is modified to maximize the clear aperture size through the transport line. For each beam splitter, the back surface is antireflection coated while the front surface is coated with appropriate reflection
TABLE I. Laser power received at each measurement station in the simultaneous profile scan system. The second column shows the reflection ratio of individual pickup optics used in each measurement station. The third and fourth columns show the light power received at each measurement station when all pickup optics are in place. The difference between calculated and measured numbers is due to the insertion of beam samplers in the LTL and the discrepancy between the actual mirror reflectivity and its nominal value.

Pickup Individual mirror Receiving power Receiving power mirror \# reflectivity (\%) ratio (calculated) ratio (measured)

\begin{tabular}{lrrr}
\hline CM01 & 100 & 11.7 & 8.9 \\
CM02 & 50 & 11.7 & 11.4 \\
CM03 & 33 & 11.7 & 9.3 \\
CM04 & 25 & 11.7 & 11.6 \\
CM12 & 20 & 11.7 & 12.7 \\
CM13 & 20 & 14.6 & 13.8 \\
CM14 & 10 & 8.1 & 10.2 \\
CM15 & 10 & 9.0 & 8.7 \\
CM32 & 10 & 10.0 & 8.2 \\
\hline \hline
\end{tabular}

ratios. The reflection ratios are properly arranged so that each measurement station receives similar light power when all pickup optics (mirrors/beam splitters) and beam samplers (for image monitoring) are in place. Table I lists the reflection ratios of individual pickup optics, the calculated and measured laser power (expressed as the ratio between the light power received by pickup optics and the laser output power) received at each measurement station.

The laser beam passes through a focusing lens before entering the vacuum chamber. The laser beam diameters on the vacuum window (after the focusing lens) were measured at each measurement station and the numbers vary from $15 \mathrm{~mm}$ (around CM12-15) to nearly $30 \mathrm{~mm}$ (at CM32). The corresponding laser fluence (intensity) on the vacuum windows are within a range of $0.05-0.5 \mathrm{~J} / \mathrm{cm}^{2}$. We have also calculated the maximum laser fluence that can be possibly delivered on the vacuum windows by assuming the maximum laser beam power to be used at the "worst" collimation condition, i.e., the laser beam is accidentally focused on one vacuum window. The maximum laser fluence is calculated to be $3 \mathrm{~J} / \mathrm{cm}^{2}$. Meanwhile, we experimentally studied the optical breakdown of the vacuum window and found out that the laser fluence thresholds are $\sim 30 \mathrm{~J} / \mathrm{cm}^{2}$ for coating burning and $\sim 90 \mathrm{~J} / \mathrm{cm}^{2}$ for substrate damage. Therefore, we can conclude that the maximum laser fluence on the vacuum windows of all laser wire stations is an order of magnitude below the optical breakdown threshold of the vacuum windows while the operational laser fluence is almost another order of magnitude lower.

Maintaining a high spatial (pointing) stability of the laser beam is critical to ensure the laser beam be intercepted by each pickup optics and therefore to perform a 


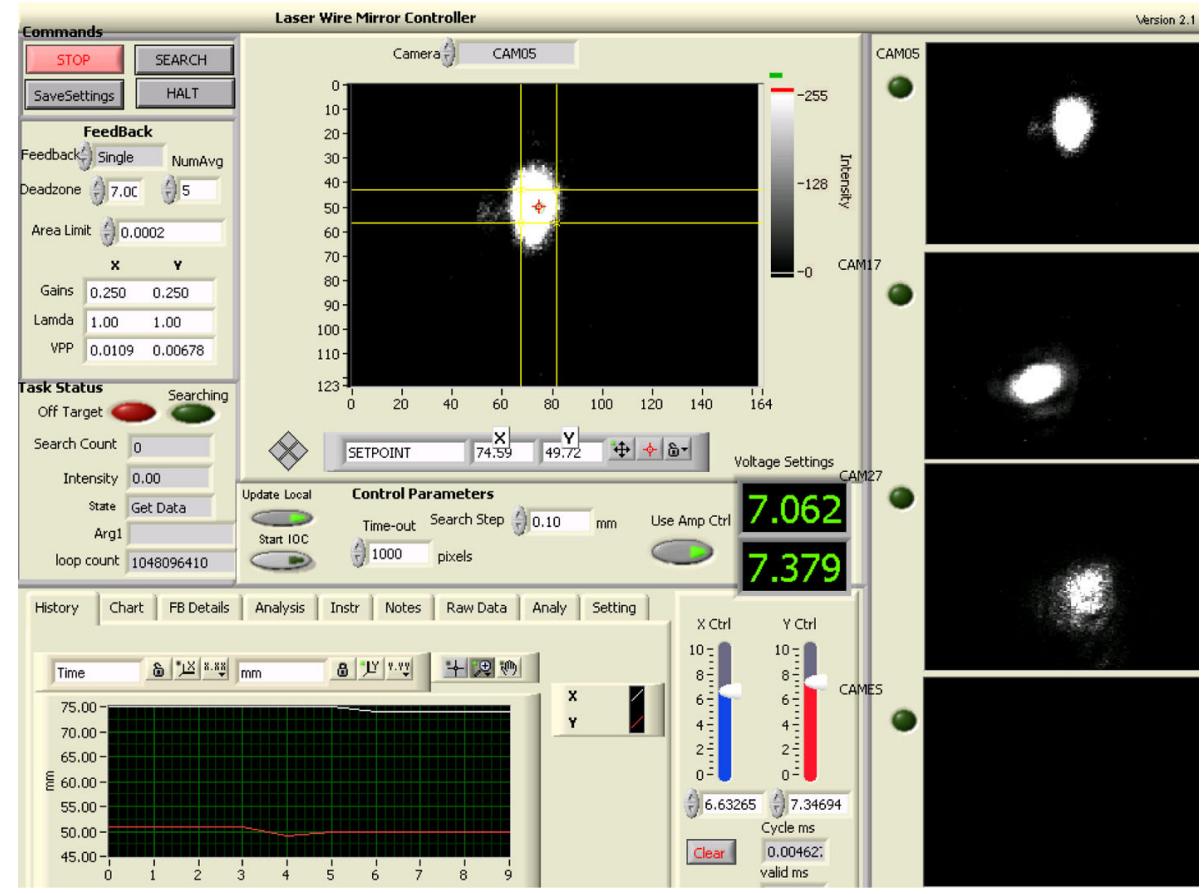

FIG. 3. Feedback-based laser beam position stabilization program. Beam positions are monitored by image sensors (Ethernet cameras) at three locations: CAM27, CAM17, and CAM05 which represent cameras installed at CM27, CM17, and CM05, respectively. Feedback is conducted based on beam positions on one or multiple image sensors.

valid profile scan. This is especially important in the case of simultaneous profile scanning as the effective aperture of the transport line becomes smaller when all pickup optics are in place. We have implemented an active stabilization scheme by steering the laser with an actuatordriven mirror installed in the laser room [11]. Figure 3 shows the laser beam pointing stabilization platform. The laser beam position can be set on either the camera installed at CM17 (referred to as CAM17) or the camera installed at CM05 (CAM05). By using feedback, the achieved laser beam stability at low frequencies is better than $5 \mu \mathrm{rad}$. This corresponds to only $\pm 1.25 \mathrm{~mm}$ of the beam position variation at the furthest measurement station, which guarantees the entire laser beam is well within the apertures of the pickup and scanning optics.

\section{Phase adjustment for simultaneous scan}

The baseline $\mathrm{H}^{-}$beam of the SNS accelerator has a $1 \mathrm{~ms}$ pulse length and a repetition rate of $60 \mathrm{~Hz}$. To match the configuration of the SNS accumulation ring, the 1-ms long $\mathrm{H}^{-}$macropulse is chopped into "minipulses" of $\sim 650$-ns duration with a period of $\sim 950 \mathrm{~ns}$. Each of the above minipulses is further bunched into $402.5 \mathrm{MHz} / 50 \mathrm{ps}$ "micropulses" by the SNS RFQ before propagating to the linac. The firing of the laser is locked to a precursor signal (timing signal) for the macropulse. As the laser pulse width is about $7 \mathrm{~ns}$, a single laser pulse will "illuminate" 3 micropulses of the ion beam. The phase delay between laser and ion beam triggers needs to be properly adjusted so that the laser pulse will not fall in the gap between minipulses, a task that can be usually fulfilled without difficulty in the case of single station scan. In the case of simultaneous scan, however, since the ion and laser beams travel in the opposite directions and the $\mathrm{H}^{-}$beam accelerates through SCL, it is not trivial to ensure that a single laser pulse will encounter the ion pulses at all measurement stations. Therefore, a careful optimization of the phase relationship between the laser pulse and the ion pulses is needed.

We analyzed the time of flight of the ion beam along the SCL based on the measured energies of the $\mathrm{H}^{-}$beam. Figure 4 shows the propagation of both the light beam and the ion beam in a temporal-space domain. The space covers from the beginning to the end of the SCL. Each vertical bar represents a minipulse ( $\sim 650 \mathrm{~ns}$ long) of the $\mathrm{H}^{-}$beam at one measurement station. Note that, as the $\mathrm{H}^{-}$ beam gains speed (energy) from acceleration, its trajectory forms a quadratic curve along the propagation as shown in Fig. 4. Three consecutive minipulses are shown in the figure with the spacing equal to the period $(\sim 950 \mathrm{~ns})$ of the minipulse. The straight line represents the trajectory of the laser pulse propagating from the end to the beginning of the SCL. We found that there exists a small time window ( $\sim 30 \mathrm{~ns})$ within which the laser pulse encounters the $\mathrm{H}^{-}$ beam at all nine measurement locations. Obviously, the laser pulse interacts at different segments, or even a completely different minipulse of the $\mathrm{H}^{-}$beam at different locations. In the experiment, we adjusted the delay time to avoid the beginning and end of minipulses which typically have a rise time of $\sim 15 \mathrm{~ns}$. 


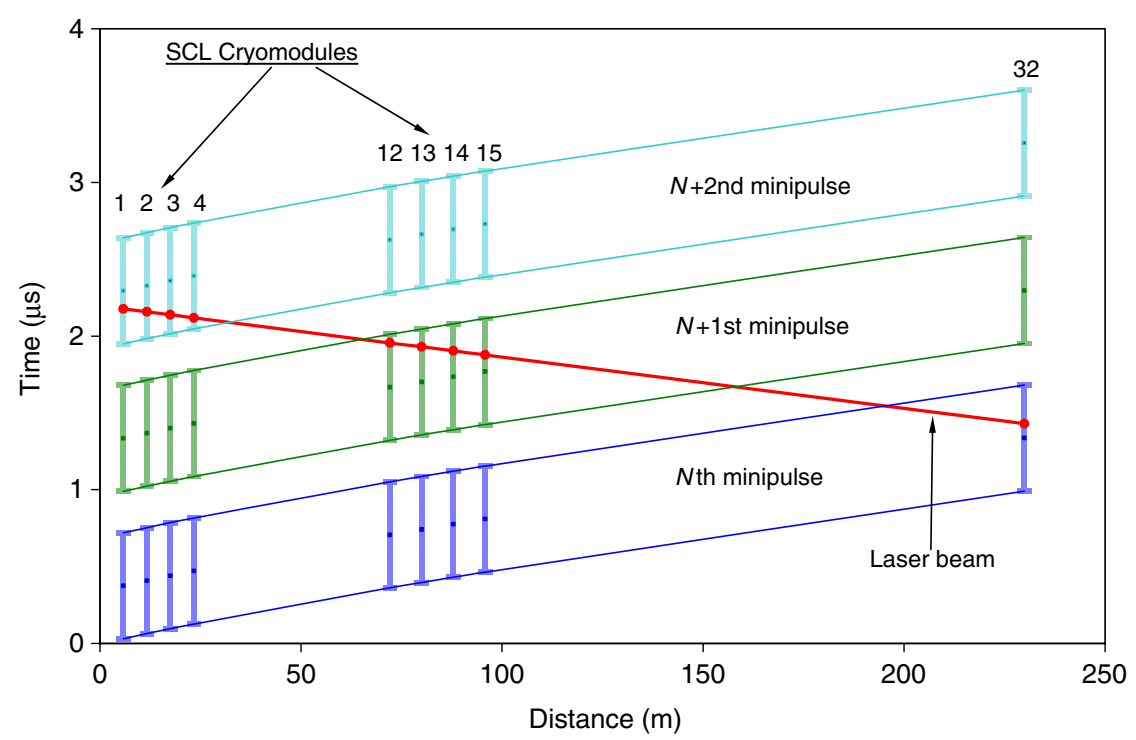

FIG. 4. Temporal-space representation of the laser pulse and ion beam's minipulses along the SCL. Distance is from the beginning of SCL. Laser pulse and ion pulses are traveling in opposite directions.

\section{MEASUREMENT RESULTS}

\section{A. Signal detection and processing}

The interaction of the $\mathrm{H}^{-}$pulses with the intense laser pulse liberates significant amounts of electrons from the ion beam (typically a few percent of the ions over the laser pulse period). The photodetached electrons are deflected in the vertical direction by a $\mathrm{C}$-shape dipole magnet [8] (collecting magnet) installed right after the interaction point. The electrons are then detected by a Faraday cup installed next to the collection magnet. The electron collecting magnet also induces a minor deflection of the ion beam which will cause some beam loss down stream. Therefore a specially designed dipole correcting magnet with the same magnetic field but in the opposite polarity is installed in the proximity of the electron collecting magnets to compensate the ion beam trajectory [9]. The correcting magnet is powered in series with the collecting dipoles from the same power supply and thus provides an automatic compensation at all magnet currents. Occasionally, we still observe some beam loss on certain sensitive beam loss monitors even with the correcting magnet turned on. In these cases, we localize the orbit distortion by using vertical dipole correctors in the downstream.

The electron collector output passes through a coaxial cable to the signal processing electronics located in the klystron gallery. Each set of signal processing electronics consists of a low-level rf circuit with two stages of amplification and a low pass filter. The amplifiers have a $>1 \mathrm{GHz}$ bandwidth while the low pass filters have a cutoff frequency of $50 \mathrm{MHz}$. The processed signal after the lowlevel $\mathrm{rf}$ circuit is typically a negative pulse with a pulse width of about $17 \mathrm{~ns}$. We calculated that $1 \mathrm{mV}$ in amplitude corresponds to about $0.03 \mathrm{pC}$ of electron charge released through photodetachment.
The processed electron signal is finally sent to an analogto-digital converter (ADC) (ACQIRIS DP235) located in the laser wire control computer for analysis and profile scan with a customized program developed in LabVIEW. The digitizer acquires the data at a sampling rate of $500 \mathrm{MS} / \mathrm{s}$ with a bandwidth of $500 \mathrm{MHz}$. Since each computer receives a time reference from the network, the location of the expected electrical signal can be found with high accuracy by calculating the propagation times of light and electrons. To increase the signal-to-noise ratio, the signal processing is performed over $\pm 100 \mathrm{~ns}$ of the anticipated position. Either the amplitude or the area of integration of the electron signal will correspond to the number of electrons detached by the laser pulse. It was verified that both methods result in an identical beam profile.

Each laser wire measurement station has a separate computer that hosts a data acquisition ADC, controls four linear motors and a flip mirror in the scan box, receives timing reference from the network to locate the signal, and performs the data analysis and profile visualization. The pickup mirrors in the transfer line are controlled by a different set of computers. All laser wire computers are accessed through the SNS accelerator network.

\section{B. Data analysis}

The profile scan time consists of two major contributions: the motion of the stepper motor and the data acquisition. To achieve a high scan accuracy, we currently move the motor at a relatively low speed so that it takes about $0.5 \mathrm{~s}$ per step (regardless of the step size). On the other hand, the data acquisition/analysis takes less than $30 \mathrm{~ms}$ per sample so it finishes before the next laser pulse arrives. To take the advantage of the fast data processing compared to the motion of the scanning motor, we usually conduct 
multiple (15-25) measurements and thus obtain multiple samples at each scanning position $x_{i}$. The averaged value $y_{i}$ over multiple samples is taken as the measured data of the profile while the standard deviation $\sigma_{i}$ is also calculated for error analysis. Finally, the measured data set $\left(x_{i}, y_{i}\right)(i=$ $1,2, \ldots, N)$ is fit with a Gaussian function

$$
y(x ; \vec{a})=a_{1} \exp \left[-\frac{\left(x-a_{2}\right)^{2}}{2 a_{3}^{2}}\right]+a_{4} .
$$

Here, $i$ represents the scanning step number, $x$ is the position, $y$ is the measurement data averaged over multiple samples, and $\vec{a}=\left[a_{1}, a_{2}, a_{3}, a_{4}\right]^{T}$ is the parameter set of the Gaussian function. The fitting is automatically started even before the entire measurement is completed. In particular, for the multiple measurement case, we use a leastsquares fitting method to optimize the coefficients, in which the quantity

$$
\chi^{2}=\sum_{i=1}^{N}\left[\frac{y_{i}-y\left(x_{i} ; a_{1}, a_{2}, a_{3}, a_{4}\right)}{\sigma_{i}}\right]^{2}
$$

is minimized. From the fit, we obtain the Gaussian function parameters including the amplitude $a_{1}$, center position $a_{2}$, beam size $a_{3}$, and offset level $a_{4}$. Furthermore, using the error analysis described in the Appendix and Ref. [12], we compute the expected variance of each parameter in the Gaussian function using the measured standard deviation $\sigma_{i}$ at each position.

\section{Software platform for simultaneous profile scan}

The laser wire software was originally implemented on the LabVIEW platform. To facilitate its operational use at SNS, the laser wire user interface screens were changed to EDM for EPICS. This allowed them to be integrated into the standard operating platform of the SNS beam instruments. Figure 5 shows an EDM operator screen of the laser wire system. From this screen, the user can pick one, multiple, or all nine laser wire profile monitors at the same time. Figure 6 shows an EDM screen of individual profile measurements station. From this page, the user can select the minipulse number of the ion beam, number of measurements at each point, scanning range, and step size. The user can conduct simultaneous scans by simply clicking "Laser wire scan!" at each measurement station screen since all scanning motors are separately addressed. Because of the detection scheme limitation, however, the user has to choose either the horizontal or vertical axis to scan. The platform also provides estimated parameter values, time needed to accomplish the scan, and the time stamp information for each measured profile. The bottom button "Expert" allows experienced users the ability to perform advanced configuration and data analysis such as the adjustment of timing, moving stages manually, and investigating raw signals at different situations.

\section{Beam profiles and parameters of simultaneous scan}

The simultaneous profile scan system was commissioned in the Spring run of 2012. Measurements were conducted on $60 \mathrm{~Hz}$ neutron production beams with an average power of up to $1 \mathrm{MW}$. Figure 7 shows a threedimensional representation of a total nine pairs of the $\mathrm{H}^{-}$ beam profiles obtained from two (i.e. horizontal and vertical) simultaneous scans. The profiles are plotted in the $X Y$ plane and are placed in the order of the beam propagation in the SCL. The calculated beam parameters with the estimated variances are summarized in Table II. Using the estimated beam size [ $a_{3}$ in Eq. (1)], we also drew a two-dimensional distribution of the $\mathrm{H}^{-}$beam at each measurement station.

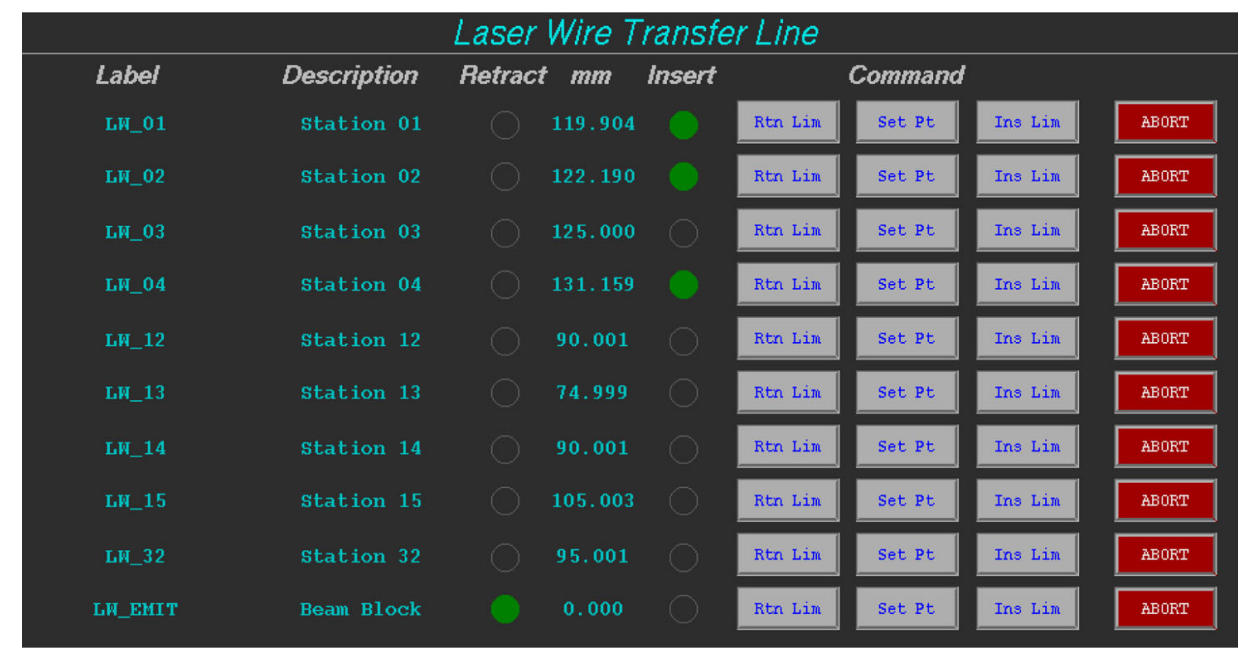

FIG. 5. Extensible display manager (EDM) screen of the laser wire transfer line control for simultaneous scans. The user can set up positions of all pickup optics separately. The lowest button in the list, LW_Emit, stands for the HEBT laser emittance measurement system which measures the transverse emittance of the ion beam using the same light source [9]. The laser emittance measurement setup is not included in the present simultaneous scan system. 


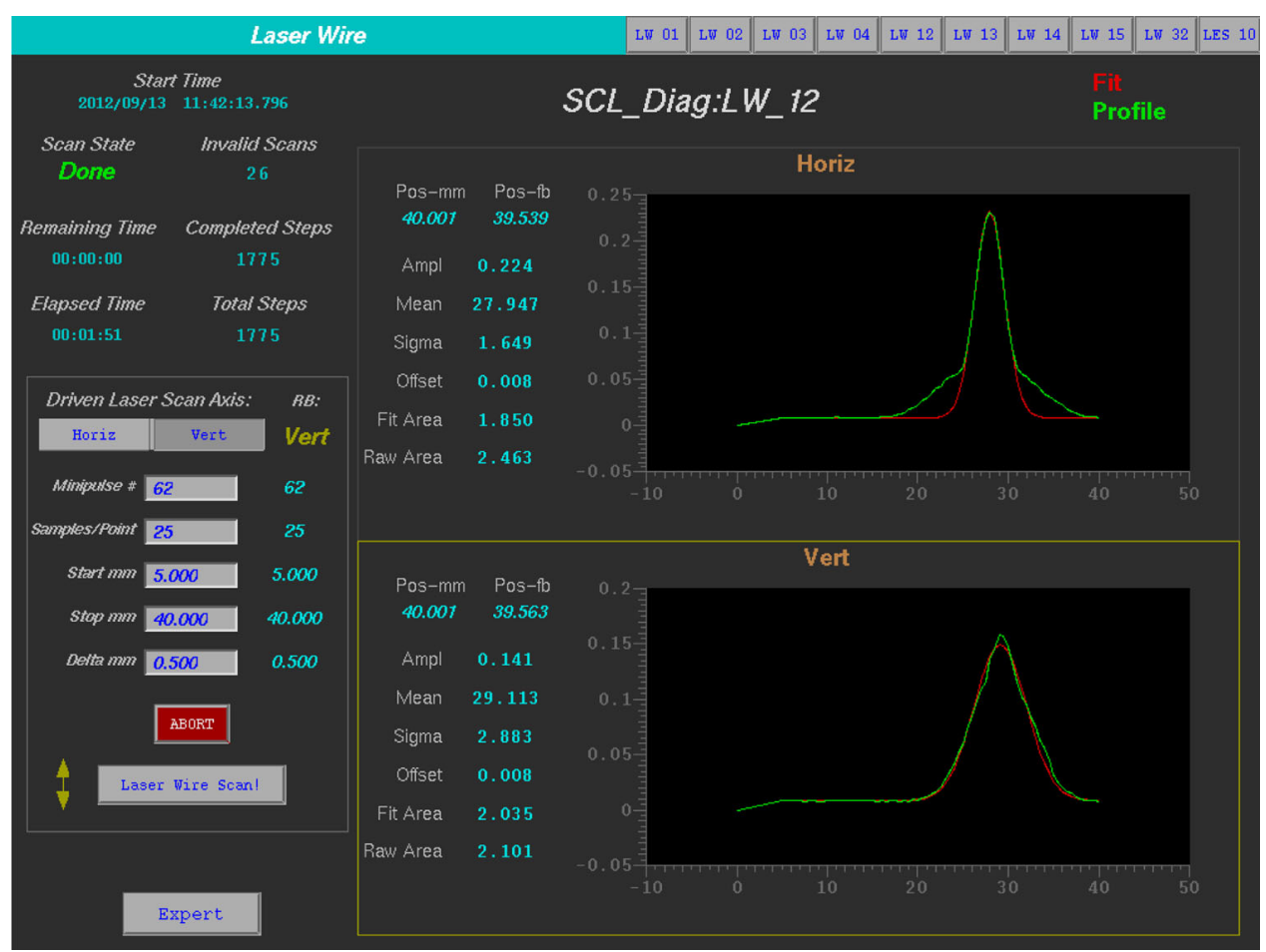

FIG. 6. An example of EDM screen of an individual measurement station (at CM 12 of SCL). The unit of the profile axes is [mm]. The top menu shows all available measurement stations. A new window will be opened when a different measurement station is selected. LES10 is the HEBT laser emittance measurement system which measures the transverse emittance of the ion beam using the same light source [9].

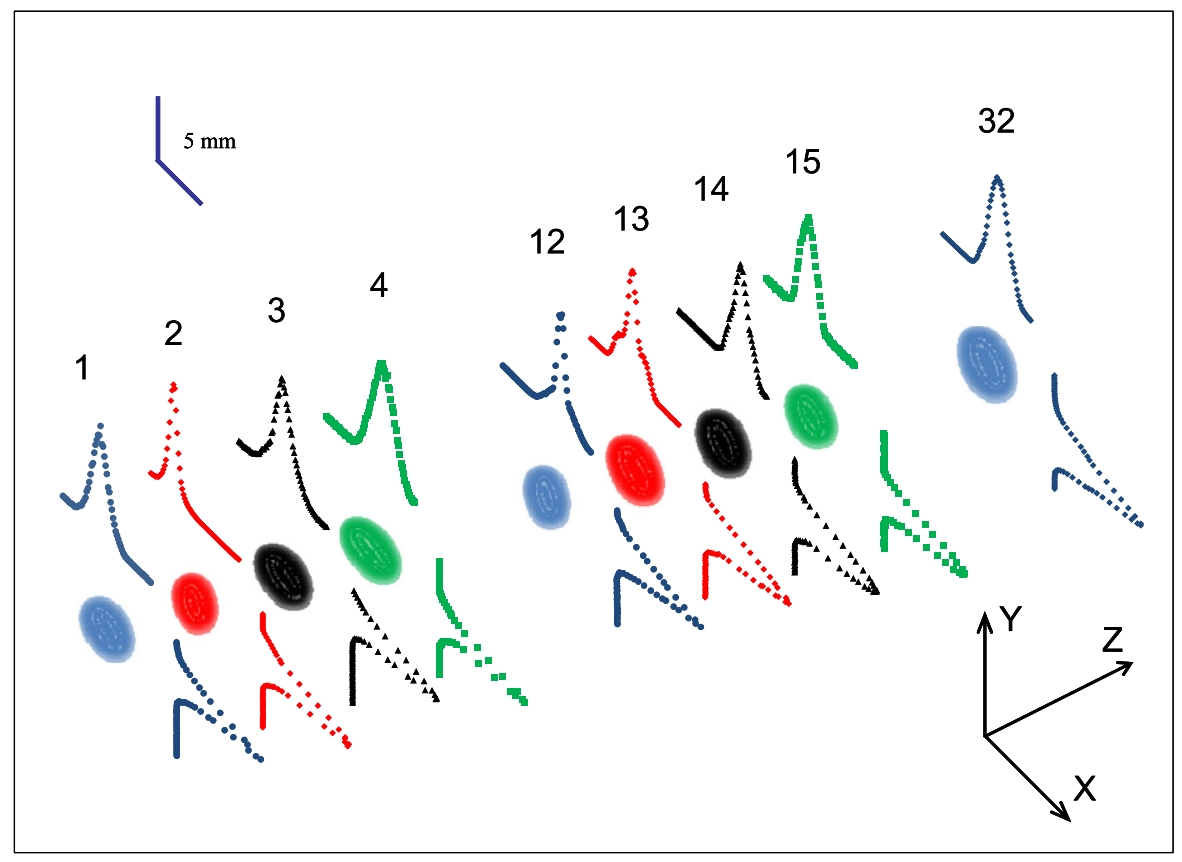

FIG. 7. All nine pairs of profiles simultaneously measured by the laser wire system. The measurement takes about 2 minutes for each axis. Numbers represent cryomodule in the SCL. The $\mathrm{H}^{-}$beam is propagating along the $Z$ direction. Profiles are plotted in the $X Y$ plane with horizontal profiles on the top. All profiles are plotted with the same scale in space. Scale bars represent 5 mm. Each elliptic shape represents the distribution of the $\mathrm{H}^{-}$beam with the measured beam size. 
TABLE II. Measured beam parameters and fitting performance with Gaussian function. $\mathrm{R}^{2}$ is the square of the correlation coefficient between measured and modeled values.

\begin{tabular}{|c|c|c|c|c|c|}
\hline Location & $\begin{array}{l}\text { Amplitude } \\
a_{1}(\mathrm{mV})\end{array}$ & $\begin{array}{l}\text { Beam center } \\
a_{2}(\mathrm{~mm})\end{array}$ & $\begin{array}{c}\text { Beam size } \\
a_{3}(\mathrm{~mm})\end{array}$ & $\begin{array}{c}\text { Offset } \\
a_{4}(\mathrm{mV})\end{array}$ & $\mathrm{R}^{2}$ \\
\hline $1 \mathrm{H}$ & $112 \pm 2$ & $19.747 \pm 0.046$ & $3.360 \pm 0.044$ & $7.5 \pm 0.2$ & 0.995 \\
\hline $1 \mathrm{~V}$ & $133 \pm 3$ & $29.397 \pm 0.035$ & $2.492 \pm 0.032$ & $7.7 \pm 0.2$ & 0.980 \\
\hline $2 \mathrm{H}$ & $244 \pm 4$ & $13.848 \pm 0.019$ & $2.085 \pm 0.017$ & $6.0 \pm 0.1$ & 0.963 \\
\hline $2 \mathrm{~V}$ & $188 \pm 2$ & $26.359 \pm 0.021$ & $2.787 \pm 0.019$ & $5.2 \pm 0.1$ & 0.983 \\
\hline $3 \mathrm{H}$ & $89 \pm 2$ & $22.617 \pm 0.061$ & $3.885 \pm 0.061$ & $8.8 \pm 0.3$ & 0.983 \\
\hline $3 \mathrm{~V}$ & $182 \pm 2$ & $32.279 \pm 0.027$ & $2.500 \pm 0.023$ & $8.3 \pm 0.2$ & 0.997 \\
\hline $4 \mathrm{H}$ & $143 \pm 1$ & $27.266 \pm 0.039$ & $4.422 \pm 0.039$ & $7.6 \pm 0.3$ & 0.998 \\
\hline $4 \mathrm{~V}$ & $262 \pm 4$ & $22.827 \pm 0.021$ & $2.091 \pm 0.019$ & $9.4 \pm 0.2$ & 0.978 \\
\hline $12 \mathrm{H}$ & $156 \pm 3$ & $27.979 \pm 0.031$ & $2.611 \pm 0.033$ & $9.9 \pm 0.2$ & 0.899 \\
\hline $12 \mathrm{~V}$ & $134 \pm 2$ & $29.355 \pm 0.034$ & $3.108 \pm 0.035$ & $8.0 \pm 0.3$ & 0.990 \\
\hline $13 \mathrm{H}$ & $129 \pm 2$ & $21.311 \pm 0.036$ & $3.786 \pm 0.039$ & $8.3 \pm 0.3$ & 0.906 \\
\hline $13 \mathrm{~V}$ & $149 \pm 2$ & $28.611 \pm 0.034$ & $3.316 \pm 0.032$ & $7.9 \pm 0.3$ & 0.996 \\
\hline $14 \mathrm{H}$ & $109 \pm 1$ & $29.648 \pm 0.037$ & $3.269 \pm 0.038$ & $7.3 \pm 0.2$ & 0.992 \\
\hline $15 \mathrm{H}$ & $73 \pm 1$ & $21.376 \pm 0.048$ & $3.047 \pm 0.050$ & $7.7 \pm 0.3$ & 0.996 \\
\hline $15 \mathrm{~V}$ & $92 \pm 1$ & $21.171 \pm 0.037$ & $2.670 \pm 0.039$ & $7.9 \pm 0.3$ & 0.998 \\
\hline $32 \mathrm{H}$ & $95 \pm 1$ & $27.073 \pm 0.020$ & $3.830 \pm 0.018$ & $2.7 \pm 0.1$ & 0.999 \\
\hline $32 \mathrm{~V}$ & $106 \pm 1$ & $20.353 \pm 0.021$ & $3.616 \pm 0.017$ & $2.2 \pm 0.1$ & 0.988 \\
\hline
\end{tabular}

Figure 8 is a snapshot recorded from the SNS beam status broadcast channel (available publicly [13]) on the day when the simultaneous profile measurement (Fig. 7) was performed. The neutron production beam shows a stable output power within the measurement time window, which clearly verifies the nonintrusive nature of the laser wire diagnostics.

The dependence of the profile measurement performance on laser and ion beam parameters has been both theoretically and experimentally investigated in previous work $[8,10]$. Specifically, we have demonstrated that the measured profile shape, beam size, and beam center position showed very little variation against changes of the laser power [10]. It was also verified from previous

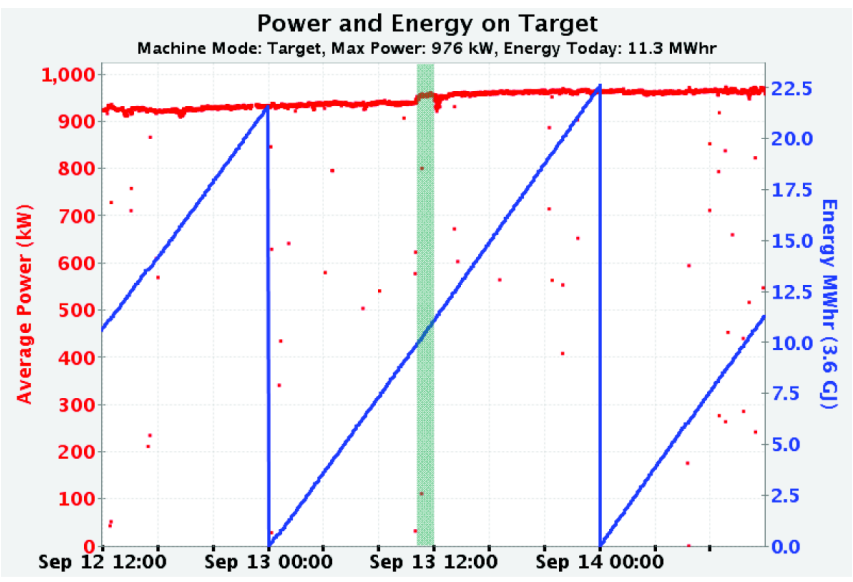

FIG. 8. A snapshot of SNS beam status around September 13, 2012. The time window for the laser wire based simultaneous profile measurement is indicated with the shadowed region. experiments that there was no significant (mini) pulse-topulse dependence in the profile parameters [8]. Therefore, we can conclude that the simultaneous profile scan will result in reliable profile measurements despite the variation of laser power and minipulse positions occurring at the different measurement stations.

The combination of Fig. 7 and Table II provides a highly intuitive and informative picture of the $\mathrm{H}^{-}$beam propagation along the acceleration path. For example, one can conveniently track the variation of the $\mathrm{H}^{-}$beam size and shape or the development of the beam halo along the SCL based on Fig. 7. A subtle change of the beam parameters including the beam size and position in the SCL can be more easily detected from the "global" picture of all profiles. As shown in the Fig. 6, a full scan with 25 samples at each measurement point only takes about 2 minutes to finish. Therefore, an illustration of 18 profiles such as Fig. 7, only takes as few as 4 minutes to update. An equivalent EDM screen of Fig. 7 will be implemented as a part of the software platform for the simultaneous profile scan system. This will provide a powerful tool for accelerator operators and physicists to monitor and/or tune the beam parameters. Currently, a SCL modeling study using the simultaneous profile scan system is being pursued.

\section{CONCLUSION}

The SNS laser wire system is among the very few operational laser based nonintrusive beam diagnostics systems implemented in accelerator facilities. We have demonstrated a simultaneous hydrogen ion beam profile scan for the first time using the upgraded SNS laser wire system. The parallel scan allows a simultaneous profile 
measurement of the neutron production hydrogen ion beam at nine different locations along the superconducting linac of SNS. The measurement has been conducted on $1 \mathrm{MW}$, neutron production ion beam. This paper summarizes the system development and the commissioning results of the simultaneous profile scan. The laser wire based simultaneous profile scan system provides an unprecedented diagnostics tool for accelerator operators and physicists by measuring beam profiles and beam parameters with high speed, high accuracy, and in a nonintrusive manner.

\section{ACKNOWLEDGMENTS}

The authors thank A. Webster and J. Diamond for the technical help. Helpful discussions with A. Shishlo and C. Allen are acknowledged. ORNL is managed by UTBattelle, LLC, under Contract No. DE-AC05-00OR22725 for the U.S. Department of Energy. This work was performed under the auspices of the Department of Energy, Office of Science/Basic Energy Sciences.

\section{APPENDIX: ERROR ESTIMATION OF ION BEAM PARAMETERS BASED ON SINGLE ROUND MULTISAMPLE MEASUREMENT}

According to Ref. [12], for a nonlinear fitting function such as the Gaussian function, the error estimation is performed based on an assumption that if parameter vector $\vec{a}$ is sufficiently close to the nominal value $\vec{a}_{0}$ (obtained from the least-squares fitting), then $y(x ; \vec{a})$ in Eq. (1) may still be expressed as a linear function of the parameter deviation,

$$
\begin{aligned}
y(x ; \vec{a}) & =y\left(x ; \vec{a}_{0}\right)+\left.\sum_{j=1}^{M} \frac{\partial y(x ; \vec{a})}{\partial a_{j}}\right|_{\vec{a}_{0}}\left(a_{j}-a_{0 j}\right) \\
& =y\left(x ; \vec{a}_{0}\right)+\left(\vec{a}-\vec{a}_{0}\right) \vec{d}\left(x ; \vec{a}_{0}\right),
\end{aligned}
$$

where $\vec{d}=\left[d_{1}, d_{2}, d_{3}, d_{4}\right]^{T}$ is a derivative vector and in our case has the following expression:

$$
\begin{gathered}
d_{1}\left(x ; \vec{a}_{0}\right)=\left.\frac{\partial y(x ; \vec{a})}{\partial a_{1}}\right|_{\vec{a}_{0}}=\exp \left[-\frac{\left(x-a_{02}\right)^{2}}{2 a_{03}^{2}}\right], \quad \text { (A2) } \\
d_{2}\left(x ; \vec{a}_{0}\right)=\left.\frac{\partial y(x ; \vec{a})}{\partial a_{2}}\right|_{\vec{a}_{0}}=a_{01} \exp \left[-\frac{\left(x-a_{02}\right)^{2}}{2 a_{03}^{2}}\right] \frac{x-a_{02}}{a_{03}^{2}}, \\
\text { (A3) } \\
d_{3}\left(x ; \vec{a}_{0}\right)=\left.\frac{\partial y(x ; \vec{a})}{\partial a_{3}}\right|_{\vec{a}_{0}}=a_{01} \exp \left[-\frac{\left(x-a_{02}\right)^{2}}{2 a_{03}^{2}}\right] \frac{\left(x-a_{02}\right)^{2}}{a_{03}^{3}}, \\
d_{4}\left(x ; \vec{a}_{0}\right)=\left.\frac{\partial y(x ; \vec{a})}{\partial a_{4}}\right|_{\vec{a}_{0}}=1 .
\end{gathered}
$$

A derivative matrix $A$ is thus defined as

$$
A_{i j}\left(\vec{a}_{0}\right)=\frac{d_{i j}\left(\vec{a}_{0}\right)}{\sigma_{i}} .
$$

The variance of each estimated parameter $a_{j}$ is then calculated from

$$
\sigma_{a_{j}}^{2}=C_{j j}
$$

with

$$
C=\left(A^{T} A\right)^{-1} .
$$

We computed the variance of the fit function, averaged over all variables and data points, $\bar{\sigma}_{y}^{2}=$ $\frac{1}{N} \sum_{i=1}^{N} \sum_{j=1}^{M} \sum_{k=1}^{M} C_{j k}\left(\vec{a}_{0}\right) d_{j}\left(x_{i} ; \vec{a}_{0}\right) d_{k}\left(x_{i} ; \vec{a}_{0}\right)$ and compared it with the direct measurement error $\bar{\sigma}_{i}^{2}=\frac{1}{N} \sum_{i=1}^{N} \sigma_{i}^{2}$. The ratio $\bar{\sigma}_{y}^{2} / \bar{\sigma}_{i}^{2}$ turns out to be close to the value $M / N(M=4$ is the number of fitting parameters and $N$ is the number of measurement points). This implies that the variance of the measurement object is reduced by the ratio $M / N$ as a result of the fitting. Therefore, a better fitting is expected if one increases the number of measurement points. We have also checked the effectiveness of the approach. To do this, we calculated the parameter variance over multiple $(>10)$ profile measurement events and compared the result with the one that is directly calculated from a single measurement (with multiple samples) using Eq. (A7). The two results agree well with each other for profiles that are well fit with Gaussian functions (typically $R^{2}>0.95$ ). However, when the profile presents a significant halo, both the fit performance and the error estimation accuracy show a large deterioration.

[1] R. C. Connolly, K. F. Johnson, D. P. Sandovai, and V. Yuan, Nucl. Instrum. Methods Phys. Res., Sect. A 312, 415 (1992).

[2] V. W. Yuan, R. C. Connolly, R. C. Garcia, K. F. Johnson, K. Saadatmand, O.R. Sander, D. P. Sandoval, and M. A. Shinas, Nucl. Instrum. Methods Phys. Res., Sect. A 329, 381 (1993).

[3] R.E. Shafer, Report No. LA-UR 98-2643.

[4] H. Sakai, Y. Honda, N. Sasao, S. Araki, Y. Higashi, T. Okugi, T. Taniguchi, J. Urakawa, and M. Takano, Phys. Rev. ST Accel. Beams 4, 022801 (2001).

[5] C. Gabor, A. Jakob, O. Meusel, J. Pozimski, J. Schäfer, and U. Ratzinger, Rev. Sci. Instrum. 73, 998 (2002).

[6] Y. Honda, N. Sasao, S. Araki, H. Hayano, Y. Higashi, K. Kubo, T. Okugi, T. Taniguchi, N. Terunuma, J. Urakawa, Y. Yamazaki, K. Hirano, M. Nomura, M. Takano, and H. Sakai, Phys. Rev. ST Accel. Beams 6, 092802 (2003).

[7] I. Agapov, G. A. Blair, and M. Woodley, Phys. Rev. ST Accel. Beams 10, 112801 (2007).

[8] Y. Liu, A. Aleksandrov, S. Assadi, W. Blokland, C. Deibele, W. Grice, C. Long, T. Pelaia, and A. Webster, Nucl. Instrum. Methods Phys. Res., Sect. A 612, 241 (2010). 
[9] Y. Liu, A. Aleksandrov, C. Long, A. Menshov, J. Pogge, A. Webster, and A. Zhukov, Nucl. Instrum. Methods Phys. Res., Sect. A 675, 97 (2012).

[10] Y. Liu, C. Long, C. Peters, and A. Aleksandrov, Appl. Opt. 49, 6816 (2010).
[11] R. Hardin, Y. Liu, C. Long, A. Aleksandrov, and W. Blokland, Opt. Express 19, 2874 (2011).

[12] P.H. Richter, TDA Progress Report No. 42-122 (1995).

[13] http://status.sns.ornl.gov/beam.jsp 